\title{
Intersectional nanotwinned diamond-the hardest polycrystalline diamond by design
}

\author{
Jianwei Xiao $\mathbb{D}^{1}$, Bin Wen $\mathbb{D}^{1 凶}$, Bo Xu $\mathbb{D}^{1}$, Xiangyi Zhang ${ }^{1}$, Yanbin Wang $\mathbb{D}^{2}$ and Yongjun Tian (iD)
}

The hardness of nanotwinned diamond (nt-diamond) is reported to be more than twice that of the natural diamond, thanks to the fine spaces between twin boundaries (TBs), which block dislocation propagation during deformation. In this work, we explore the effects of additional TBs in nt-diamond using molecular dynamics (MD) calculations and introduce a novel intersectional nanotwinned diamond (int-diamond) template for future laboratory synthesis. The hardness of this int-diamond is predicted by first analyzing individual dislocation slip modes in twinned grains and then calculating the bulk properties based on the Sachs model. Here we show that the hardness of the int-diamond is much higher than that of nt-diamond. The hardening mechanism of intdiamond is attributed to the increased critical resolved shear stress due to the presence of intersectional TBs in nt-diamond; this result is further verified by MD simulations. This work provides a new strategy for designing new super-hard materials in experiments.

npj Computational Materials (2020)6:119; https://doi.org/10.1038/s41524-020-00387-3

\section{INTRODUCTION}

Diamond is the hardest, stiffest and least compressible crystalline materials in the world. Understanding and further improving its hardness is scientifically fascinating and technologically impor$\operatorname{tant}^{1-3}$. In the past few decades, numerous efforts have been made in these directions, both experimentally and theoretically ${ }^{4-9}$. It has been demonstrated that the hardness of the diamond can be improved by refining its grain size and/or twin thickness according to the well-known Hall-Petch effect ${ }^{10,11}$. For example, nanograined diamond (ng-diamond) with grain sizes of 10-30 nm has been reported as high as 110-140 GPa in Knoop hardness, significantly higher than that of single-crystal diamond ${ }^{4,5,12}$. Nanotwinned diamond (nt-diamond) with an average twin thickness $(\lambda)$ of $5-8 \mathrm{~nm}$, synthesized by compressing onionstructured precursors ${ }^{7,8}$, is recently reported to possess Vickers hardness of $175-200 \mathrm{GPa}$, setting a new world record. Can the hardness of nt-diamond be further increased? This is a fundamental scientific question for designing new superhard materials with potentially wide-ranging implications ${ }^{13}$.

The recent molecular dynamic (MD) simulations on nt-diamond indicated that the origin of the unprecedented hardness originates from two factors: high lattice frictional stress due to the strong $\mathrm{sp}^{3} \mathrm{C}-\mathrm{C}$ bonding and high athermal stress due to Hall-Petch effect ${ }^{14}$. Experimental and theoretical studies also show that twin boundaries (TBs) can continuously harden covalent materials with decreasing twin thickness $(\lambda)$ to very small values (on the order of a few $\mathrm{nm})^{7,8,15,16}$. Therefore, for covalent materials, a practical strategy to achieve superhardness is to introduce more TBs into the microstructure. Based on this idea, a novel intersectional nanotwinned diamond (int-diamond) model is constructed by inducing intersectional TBs into nt-diamond. After analyzing dislocation slip modes in individual int-diamond grains, the hardness of bulk int-diamond is calculated based on the Sachs model ${ }^{17}$. We find that the hardness of the int-diamond is much higher than that of the nt-diamond. This result is further verified by MD simulations. This work provides a new strategy for designing new super-hard materials in future experiments.

\section{RESULTS}

Dislocation slip modes in int-diamond grains

For diamond, dominant dislocations are along the $<110\rangle$ directions slipping in the (111) plane with Burgers vector of $\frac{1}{2}$ $<110>$ for perfect dislocation and $\frac{1}{6}<112>$ for glide-set partial dislocation ${ }^{14,18}$. According to the angle between Burgers vector and dislocation line direction, there are six types of dislocations: glide-set $0^{\circ}$ perfect dislocations, glide-set $30^{\circ}$ partial dislocations, glide-set $60^{\circ}$ perfect dislocations, glide-set $90^{\circ}$ partial dislocations, shuffle-set $0^{\circ}$ perfect dislocations, and shuffle-set $60^{\circ}$ perfect dislocations ${ }^{14}$. Among them, shuffle-set $0^{\circ}$ perfect dislocation has the lowest critical resolved shear stress (CRSS) for dislocation motion and the lowest barrier strength when reacting with $\mathrm{TBs}^{14}$. Here the CRSS is defined as the threshold stress of dislocation motion, and the barrier strength is defined as the threshold stress of dislocation reaction with the TB when the activation energy reaches zero ${ }^{19}$. Therefore, at room temperature, the hardness of the diamond is primarily controlled by the behavior of shuffle-set $0^{\circ}$ perfect dislocations ${ }^{16,20}$. Therefore, in the present study, the hardness of the int-diamond will be analyzed based on the behavior of shuffle-set $0^{\circ}$ perfect dislocations.

In an int-diamond grain, two different orientation twin boundaries $\mathrm{TB}_{1}$ and $\mathrm{TB}_{2}$ coexist and interweaved, whose average twin thickness is $\lambda_{1}$ and $\lambda_{2}$, respectively (Fig. $1 a^{21,22}$. TB 1 and $\mathrm{TB}_{2}$ segment the grain into domains with four different crystallographic orientations, that is, orientations $D_{1}, D_{2}, D_{3}$, and $D_{4}$. Among these different orientations, lattices of $D_{1}$ and $D_{2}, D_{2}$ and $D_{3}, D_{3}$ and $D_{4}$, and $D_{4}$ and $D_{1}$ are mirror images across $T B_{1}, T_{2}$, $\mathrm{TB}_{1}$, and $\mathrm{TB}_{2}$, respectively. Consequently, the slip systems in an intdiamond grain can be expressed by a combination of four Thompson tetrahedra (in Fig. $1 b$ ): $A B C D, A B C D^{1}, A^{1} B C D$ and $A^{2} B C D^{1}$, which correspond to $D_{1}, D_{2}, D_{3}$, and $D_{4}$, respectively.

${ }^{1}$ Center for High Pressure Science, State Key Laboratory of Metastable Materials Science and Technology, Yanshan University, 066004 Qinhuangdao, China. ${ }^{2}$ Center for Advanced Radiation Sources, University of Chicago, Chicago, IL 60439, USA. ${ }^{凶}$ email: wenbin@ysu.edu.cn 

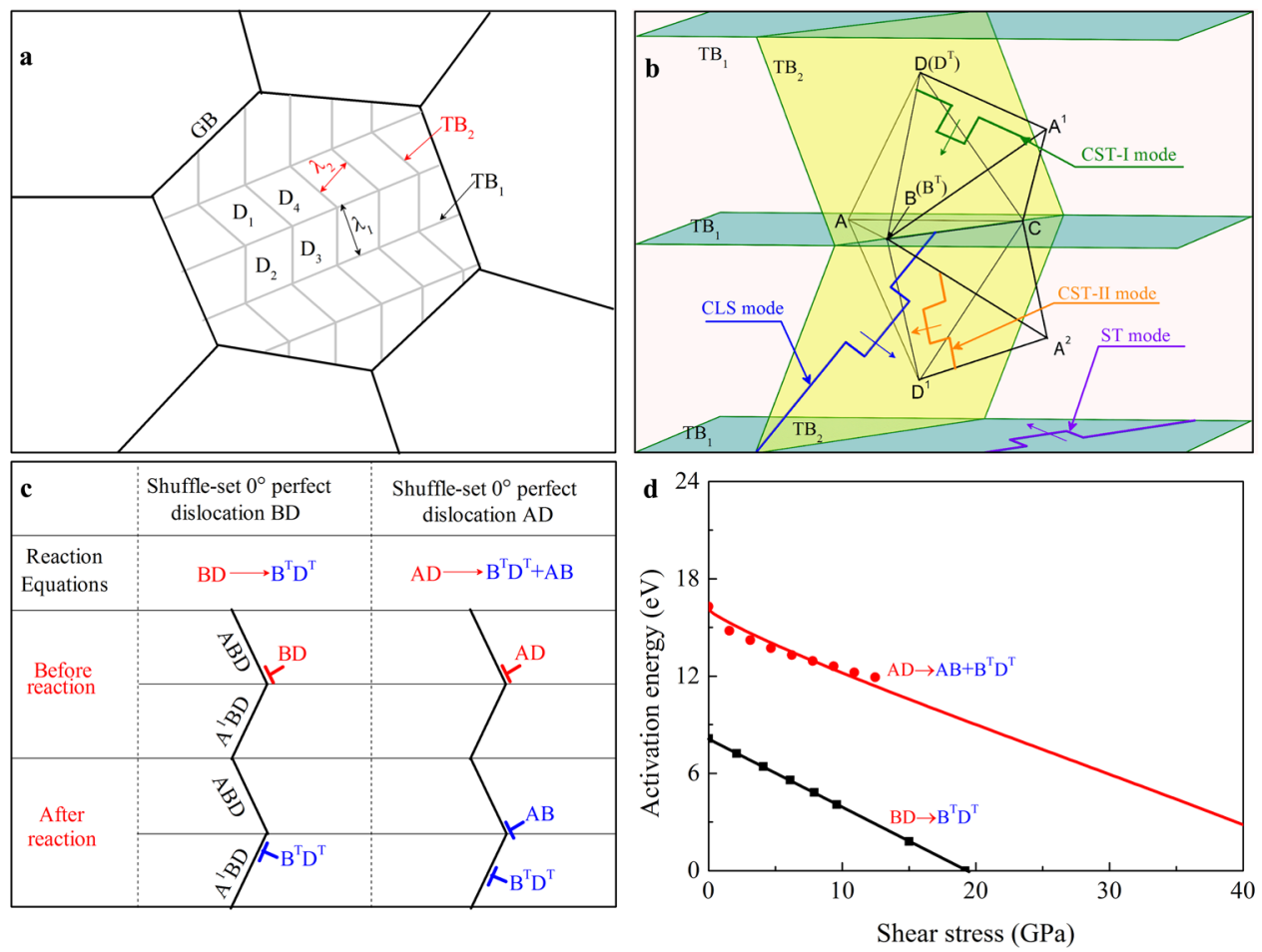

Fig. 1 Schematics of the int-diamond microstructure and its slip modes. a Structure schematic of int-diamond. Lattices of $D_{1}$ and $D_{2}, D_{2}$ and $D_{3}, D_{3}$ and $D_{4}$, and $D_{4}$ and $D_{1}$ are mirror images across twin boundary $T B_{1}, T B_{2}, T B_{1}$, and $T B_{2}$, respectively. GB is grain boundary. $\mathbf{b}$ Four dislocation slip modes in int-diamond: slip transfer mode (ST), confined layer slip mode (CLS), confined slip transfer mode I (CST-I), and confined slip transfer mode II (CST-II). c Schematic of shuffle-set screw perfect dislocation BD and AD reaction with TB when it reaches TB. d The stress-dependent activation energy of shuffle-set screw perfect dislocation BD and AD reaction with TB.

The combination of these four Thompson tetrahedra results in 39 slip systems in an int-diamond grain (Table 1). According to dislocation line directions and their slip plane orientations, the slip modes of shuffle-set $0^{\circ}$ perfect dislocations in int-diamond are divided into four types: slip transfer (ST) mode, confined layer slip (CLS) mode, confined slip transfer mode I (CST-I) and confined slip transfer mode II (CST-II) modes, all of which are schematically plotted in Fig. 1b. For ST mode, the slip planes are parallel to either $\mathrm{TB}_{1}$ or $\mathrm{TB}_{2}$, and the respective dislocation lines are located within either $\mathrm{TB}_{2}$ or $\mathrm{TB}_{1}$. For CLS mode, the slip planes are parallel to either $\mathrm{TB}_{1}$ or $\mathrm{TB}_{2}$, while the respective dislocation lines are nonparallel to $\mathrm{TB}_{2}$ or $\mathrm{TB}_{1}$. For CST-I mode, both slip planes and dislocation lines are non-parallel to any TB. For CST-II mode, the slip planes are non-parallel to any $T B$, while the respective dislocation lines are located within either $\mathrm{TB}_{1}$ or $\mathrm{TB}_{2}$. As a result, the interactions of these slip modes with TBs are different. This leads to different CRSSs for the four slip modes. For ST mode, the dislocation-TB reaction is characterized by dislocations propagating within the $\mathrm{TBs}^{23,24}$, and the corresponding CRSS is determined by the lattice friction stress and barrier strength of shuffle-set $0^{\circ}$ perfect dislocations reacting with the $\mathrm{TBs}^{25}$. For CLS mode, the dislocation motion is confined between two TBs and its CRSS can be evaluated by increased dislocation energy due to the dislocation line is left on the two TBs ${ }^{23,26}$. For CST-I mode, the shuffle-set $0^{\circ}$ perfect dislocation is confined between two TBs and then become shuffle-set $60^{\circ}$ perfect dislocation to parallel TB when it reaches TB because the initial dislocation line is nonparallel to TB. Finally, the shuffle-set $60^{\circ}$ perfect dislocation interaction with TB to propagate through TB, and its CRSS is determined by the barrier strength of shuffle-set $60^{\circ}$ perfect dislocations reacting with TBs and increased dislocation energy because produced dislocation on two TBs. For CST-II mode, although its dislocation motion is similar to that in CST-I mode, its barrier strength is determined by shuffle-set $0^{\circ}$ perfect dislocations reacting with the TB. In order to obtain CRSSs for these slip modes, barrier strengths of shuffle-set $0^{\circ}$ and $60^{\circ}$ perfect dislocations reacting with TBs must be calculated first.

Barrier strengths of dislocation reaction with TB

Shuffle-set $0^{\circ}$ and $60^{\circ}$ perfect dislocations reacting with TBs can be considered as a kink nucleation and migration process ${ }^{14,27,28}$ (Fig. 1c and Supplementary Fig. 1). The shear-stress dependent activation energy for kink nucleation and migration are calculated (detail in "Methods" section), and the results are plotted in Fig. 1d. With increasing shear stress, the activation energies for shuffle-set $0^{\circ}$ and $60^{\circ}$ perfect dislocations reacting with TB reach zero at shear stresses of 19 and $48 \mathrm{GPa}$, respectively. These stresses are considered the respective barrier strengths for shuffle-set $0^{\circ}$ and $60^{\circ}$ perfect dislocations. The twin intersecting points can provide the pinning obstacle for the slip of dislocation when the shuffleset dislocation slip along the twin plane. However, in nt-diamond, the shuffle-set dislocations slip along twin plane energetically show no advantage over those along other slip planes ${ }^{16}$. Therefore, the shuffle-set dislocation is favor to slip along the slip planes rather than twin plane, and the pinning effect of intersecting points on shuffle-set dislocation motion is neglected in this work.

\section{CRSS for ST mode}

In ST mode, dislocation motions are blocked by TBs (inset of Fig. $2 \mathrm{a})^{29}$. According to dislocation pile-up theory ${ }^{30}$, the CRSS $\left(\tau_{\text {css }}\right)$ of this mode is expressed as the following: $:^{25}$

$\tau_{\mathrm{cSS}}^{\mathrm{ST}}=\tau_{0}+\left(\frac{\tau_{\mathrm{TB}} G b}{\pi \lambda}\right)^{1 / 2}$,

where $\tau_{0}$ is lattice frictional stress; $G$ is the shear modulus; $b$ is the magnitude of the Burgers vector; $\lambda$ is twin thickness of 


\begin{tabular}{|c|c|c|c|}
\hline \multirow[t]{2}{*}{ No. } & \multirow[t]{2}{*}{ Category } & \multicolumn{2}{|l|}{ Slip system } \\
\hline & & Slip plane & Direction \\
\hline 1 & \multirow[t]{5}{*}{ ST mode } & DBC & $\mathrm{BC}$ \\
\hline 2 & & $A B C$ & $\mathrm{BC}$ \\
\hline 3 & & $A^{1} B C$ & $\mathrm{BC}$ \\
\hline 4 & & $D^{1} B C$ & $\mathrm{BC}$ \\
\hline 5 & & $A^{2} B C$ & $\mathrm{BC}$ \\
\hline 6 & \multirow[t]{10}{*}{ CLS mode } & DBC & DB \\
\hline 7 & & DBC & $\mathrm{DC}$ \\
\hline 8 & & $D^{1} B C$ & $D^{1} B$ \\
\hline 9 & & $\mathrm{D}^{1} \mathrm{BC}$ & $D^{1} C$ \\
\hline 10 & & $A^{1} B C$ & $A^{1} B$ \\
\hline 11 & & $A^{1} B C$ & $A^{1} C$ \\
\hline 12 & & $\mathrm{~A}^{2} \mathrm{BC}$ & $A^{2} B$ \\
\hline 13 & & $A^{2} B C$ & $A^{2} C$ \\
\hline 14 & & $A B C$ & $A B$ \\
\hline 15 & & $A B C$ & $A C$ \\
\hline 16 & \multirow[t]{8}{*}{ CST-I mode } & DAB & $A D$ \\
\hline 17 & & $A^{1} D B$ & $A^{1} D$ \\
\hline 18 & & $D^{1} A B$ & $A D^{1}$ \\
\hline 19 & & $A^{2} D^{1} B$ & $A^{2} D^{1}$ \\
\hline 20 & & DAC & $A D$ \\
\hline 21 & & $A^{1} D C$ & $A^{1} D$ \\
\hline 22 & & $D^{1} A C$ & $A D^{1}$ \\
\hline 23 & & $A^{2} D^{1} C$ & $A^{2} D^{1}$ \\
\hline 24 & \multirow[t]{16}{*}{ CST-II mode } & $\mathrm{DAB}$ & $A B$ \\
\hline 25 & & $A^{1} D B$ & $A^{1} B$ \\
\hline 26 & & $D^{1} A B$ & $A B$ \\
\hline 27 & & $A^{2} D^{1} B$ & $A^{2} B$ \\
\hline 28 & & DAC & $A C$ \\
\hline 29 & & $A^{1} D C$ & $A^{1} C$ \\
\hline 30 & & $D^{1} A C$ & $A C$ \\
\hline 31 & & $A^{2} D^{1} C$ & $A^{2} C$ \\
\hline 32 & & DAB & DB \\
\hline 33 & & $A^{1} D B$ & DB \\
\hline 34 & & $D^{1} A B$ & $D^{1} B$ \\
\hline 35 & & $A^{2} D^{1} B$ & $D^{1} B$ \\
\hline 36 & & DAC & $\mathrm{DC}$ \\
\hline 37 & & $A^{1} D C$ & DC \\
\hline 38 & & $D^{1} A C$ & $D^{1} C$ \\
\hline 39 & & $A^{2} D^{1} C$ & $D^{1} C$ \\
\hline
\end{tabular}

intersectional twin; $\tau_{T B}$ is the barrier strength of shuffle-set $0^{\circ}$ perfect dislocations when reacting with the TB. Both modulus and stress are in $\mathrm{GPa}$, and all length parameters are in $\mathrm{nm}$.

With $G=540 \mathrm{GPa}$ according to ref. ${ }^{14}, \tau_{T B}=19 \mathrm{GPa}$ as calculated above and $\tau_{0}=10.3 \mathrm{GPa}$ (see "Methods" section) and assuming $\lambda_{1}=\lambda_{2}=\lambda$, twin thickness-dependent CRSS for ST mode is rewritten as the following:

$\tau_{\mathrm{csS}}^{\mathrm{ST}}=10.3+28 \lambda^{-1 / 2}$,

which is plotted in Fig. 2a. This CRSS increases with decreasing twin thickness, and the trend and quantitative values are similar to that of ST mode in nt-diamond ${ }^{14}$.
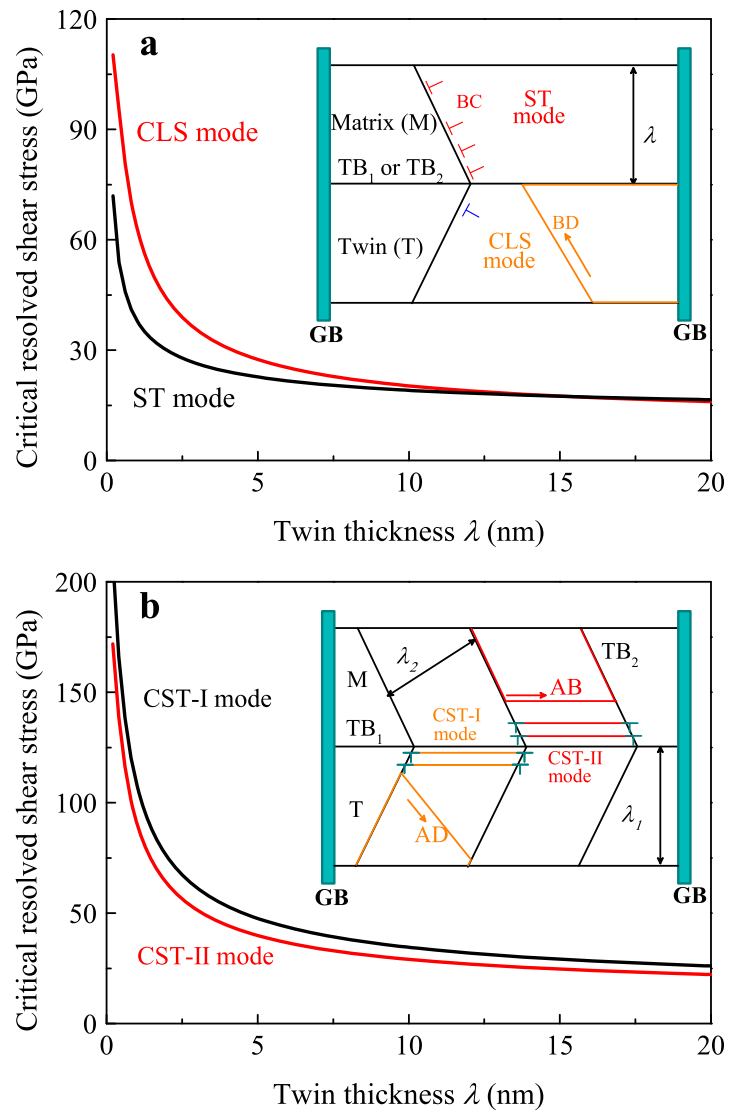

Fig. 2 Critical resolved shear stress (CRSS) for different slip modes. a Calculated CRSS of slip transfer (ST) mode and confined layer slip (CLS) mode. A dislocation pile-up model and principles of virtual work is used for ST and CLS mode, respectively. b Calculated CRSS of confined slip transfer mode I (CST-I), and confined slip transfer mode II (CST-II). For CST-I and CST-II model, it can be considered as a superposition of ST and CLS modes. The inset is schematic of shuffle-set screw perfect dislocation $A B, A D, B C$ and $B D$ reaction with twin boundary $\mathrm{TB}_{1}$ and $\mathrm{TB}_{2} . \mathrm{GB}$ is grain boundary.

CRSS for CLS mode

The CRSS for CLS mode can be calculated on the basis of the virtual work principle (Fig. 2), and it is expressed as: refs. ${ }^{25,31}$

$\tau_{\mathrm{css}}^{\mathrm{CLS}}=\tau_{0}+\frac{\mathrm{Gb}\left[1-\nu \cos ^{2}(\phi)\right]}{2 \pi(1-\nu)} \frac{\sin \theta}{\lambda} \ln \frac{\lambda a}{b}$,

where $\theta$ is the angle between the slip plane and the twin plane and $\lambda$ is twin thickness; $v$ is Possion ratio; $\phi$ is the angle between the dislocation line and the Burgers vector, $a$ is dislocation core parameter $^{23}$.

With the corresponding parameters from ref. 14 and materials parameters of the diamond (listed in Supplementary Table 1), the CRSS for CLS mode is expressed as:

$\tau_{\mathrm{css}}^{\mathrm{CLS}}=10.3+22 \frac{1}{\lambda} \ln \left(\frac{3.33 \lambda}{0.25}\right)$.

Using Eq. 4, the CRSS for CLS mode is calculated and plotted in Fig. 2a. This CRSS increases with decreasing twin thickness, and the values are similar to that of CLS mode in nt-diamond ${ }^{14}$.

\section{CRSS for CST-I mode}

In CST-I mode, dislocation motions are confined by $\mathrm{TB}_{1}$ or $\mathrm{TB}_{2}$ and blocked by $\mathrm{TB}_{2}$ or $\mathrm{TB}_{1}$, respectively. Therefore, the corresponding CRSS is affected by both the Hall-Petch effect and the confined 

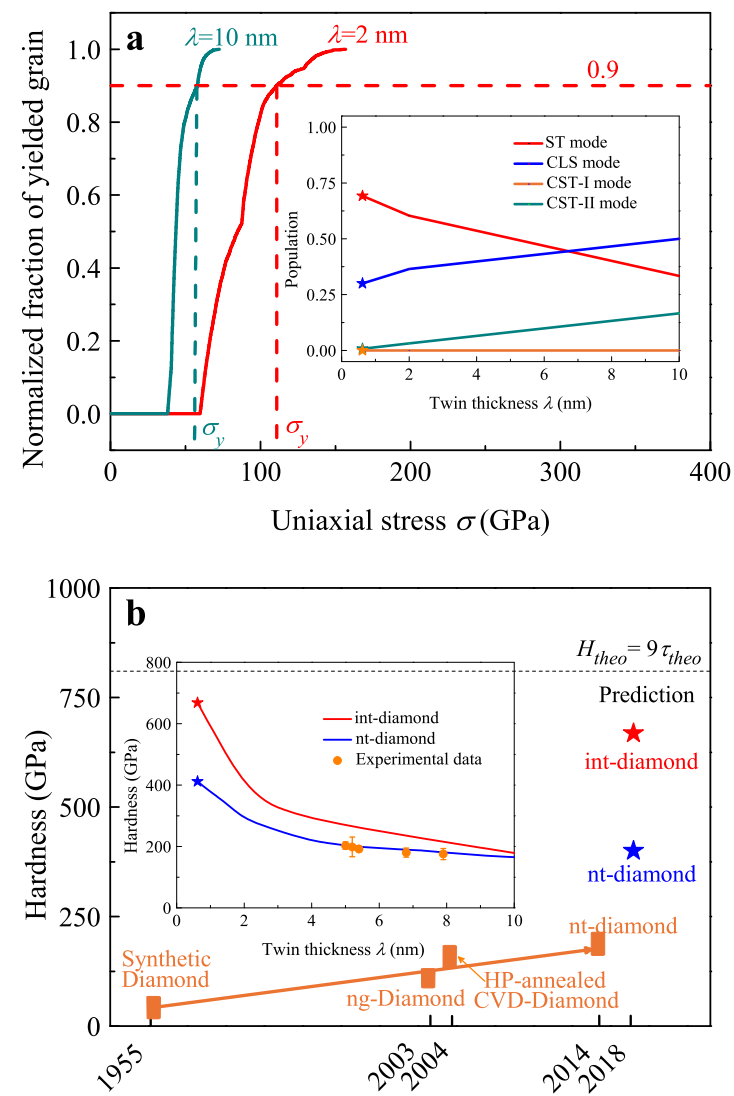

Fig. 3 Calculated hardness for int-diamond. a Fraction of yielded grains as a function of uniaxial stress. When the fraction of yielded grains reaches $90 \%$, the corresponding uniaxial stress is defined as yield stress $\sigma_{y}$. The inserted figure is the statistical fraction of different slip modes occurring in the yielded int-diamond grains. b The calculated hardness of int-diamond compared with ngdiamond and nt-diamond. The inserted figure is the twin-thicknessdependent hardness of int-diamond and nt-diamond calculated by the Sachs model, together with the comparison of experimental measurements data ${ }^{7,8}$. $H_{\text {theo }}$ is theoretical hardness of diamond calculated by the $9 \tau_{\text {theo, }}$ where the $\tau_{\text {theo }}$ is the theoretical shear strength of diamond.

layer slipping effect. To obtain CRSS, both dislocation pile-up and CLS models are used (in Fig. 2b), and the CRSS for CST-I mode is expressed as:

$\tau_{\mathrm{cSS}}^{\mathrm{CST}-1}=\tau_{0}+\left(\frac{\tau_{T B} \mathrm{~Gb}}{\pi \lambda}\right)^{1 / 2}+\frac{\mathrm{Gb}\left[1-\nu \cos ^{2}(\phi)\right]}{2 \pi(1-\nu)} \frac{\sin \theta}{\lambda} \ln \frac{\lambda a}{b}$,

Where $\tau_{0}$ is lattice frictional stress, $\tau_{\mathrm{TB}}$ is the barrier strength of shuffle-set $60^{\circ}$ perfect dislocation reacting with TB, and $v$ is the Poisson ratio. All other parameters have been defined before.

Based on the parameters of the diamond from ref. ${ }^{14}$ (listed in Supplementary Table 1), the CRSS of CST-I mode is expressed as:

$\tau_{\mathrm{CSS}}^{\mathrm{CST}-1}=10.3+45.3 \lambda^{-1 / 2}+22 \frac{1}{\lambda} \ln \left(\frac{3.33 \lambda}{0.25}\right)$.

The resulting CRSS as a function of twin thickness is plotted in Fig. 2b. This CRSS increases with decreasing twin thickness. Because the CRSS is affected by both the Hall-Petch effect and the confined layer slipping effect, it can be considered as a superposition of ST and CLS modes. At the same twin thickness condition, this CRSS is higher than that of ST and CLS modes.

\section{CRSS for CST-II mode}

Similar to CST-I mode, the CRSS for CST-II is affected by both the Hall-Petch effect and the confined layer slipping effect; therefore, the CRSS for CST-II can be expressed by Eq. 5. The difference is that in this case, $\tau_{\mathrm{TB}}$ refers to the barrier strength of shuffle-set $0^{\circ}$ perfect dislocations reacting with TB. Based on Eq. 5 and the parameters of the diamond, the CRSS for CST-II mode is expressed as:

$\tau_{\mathrm{cSS}}^{\mathrm{CST}-\|}=10.3+28 \lambda^{-1 / 2}+22 \frac{1}{\lambda} \ln \left(\frac{3.33 \lambda}{0.25}\right)$.

The CRSS of CST-II mode thus calculated is plotted in Fig. 2b. Due to the lower barrier strength of shuffle-set $0^{\circ}$ perfect dislocations, the CRSS is smaller than that of CST-I at the same twin thickness. Owing to the combined Hall-Petch and confined layer slipping effects, this CRSS is also higher than that of ST and CLS modes at the same twin thickness.

Hardness of bulk int-diamond based on the Sachs model The Sachs model is a single-slip system model for mechanical properties of polycrystalline materials, and it is a particularly effective method to investigate the yield strength of polycrystalline materials with anisotropic slip systems ${ }^{17}$. For int-diamond, dislocations in multiple twin domains change directions and slip planes in such complex manner (as shown in Figs. 1 and 2) that the yield strength cannot be evaluated using a simple Taylor model $^{32}$. Here, we model the yield strength of bulk int-diamond by considering 6000 grains on the basis of the Sachs model (see "Methods" section). Macroscopic yield strength is defined as the stress level at which $90 \%$ of the grains yield. The Vickers hardness is then assumed to be three times the compressive yield strength $^{33-37}$. The resulting hardness of int-diamond increases with decreasing twin thickness (both $\lambda_{1}$ and $\lambda_{2}$ ), and is consistently higher than that of nt-diamond with the same twin thickness $\left(\lambda_{1}\right)$ and grain size (Fig. 3b). At a twin thickness of $0.62 \mathrm{~nm}$, intdiamond reaches a hardness of $668 \mathrm{GPa}, \sim 67 \%$ higher than that of the nt-diamond $(401 \mathrm{GPa})$. These results indicate that the hardness limit for nt-diamond can be raised further by adding intersectional TBs in nt-diamond. Although the hardness limit of the intdiamond can be improved to $668 \mathrm{GPa}$, it is still below the theoretical hardness $(\sim 810 \mathrm{GPa})$ of diamond calculated by the $9 \tau_{\text {theo, }}$, where the $\tau_{\text {theo }}$ is the theoretical shear strength of diamond.

The fractions of different slip modes occurring in the yielded int-diamond grains are statistically analyzed, and the results are plotted in the insert of Fig. 3a. The fraction of grains yielded by ST mode slips increases whereas the fraction of grains yielded by CLS and CST-II mode slips decreases with decreasing twin thickness. Slips by CST-I mode are difficult to activate due to the high CRSSs, therefore the fraction of grains yielded by CST-I mode slips is essentially zero within the twin thickness range studied here. Hence, the hardness of bulk int-diamond is primarily due to slips in the ST, CLS, and CST-II modes with twin thicknesses up to $10 \mathrm{~nm}$. As the CRSS of CST-II slip is higher than that of slip mode in nt-diamond, the hardness of int-diamond is higher than that of nt-diamond ${ }^{14}$.

\section{Verification of int-diamond hardness by MD simulation}

To further confirm the calculated results by Sachs model, the yield strength of polycrystalline int-diamond is studied by using MD simulation. The calculated stress-strain curve of the int-diamond is plotted in Fig. 4. The yield strength is equal to $165 \mathrm{GPa}$ for intdiamond, 154 and $161 \mathrm{GPa}$ for nt-diamond at twin thickness of 5.5 and $1.2 \mathrm{~nm}$, and $140 \mathrm{GPa}$ for ng-diamond, respectively. Although the strain rate $\left(5 \times 10^{8} \mathrm{~s}^{-1}\right)$ in MD simulation is higher than that the experiment, these results qualitatively confirm that the yield 


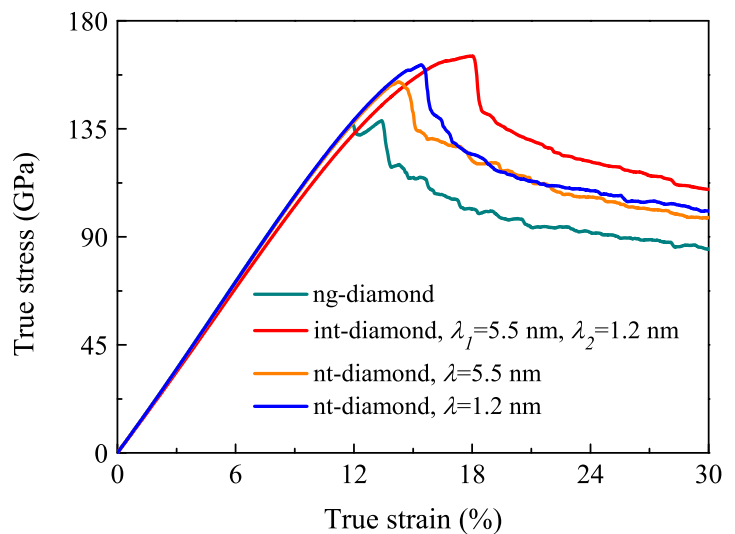

Fig. 4 Calculated stress-strain curves of int-diamond, nt-diamond, and ng-diamond. The red, yellow, blue and cyan line is the stressstrain curve of int-diamond with twin thickness $\lambda_{1}=5.5 \mathrm{~nm}$ and $\lambda_{2}=1.2 \mathrm{~nm}$, nt-diamond with twin thickness $\lambda$ of 5.5 and $1.2 \mathrm{~nm}$, and ng-diamond, respectively.

strength of the int-diamond is larger than that of the nt-diamond, and further confirm the simulated results by our Sachs model.

Applicability of the int-diamond idea to synthesis

Huang et al. ${ }^{7}$ and Tao et al. ${ }^{8}$ have shown that, with properly selected precursory materials and under carefully controlled synthesis conditions, nano twins can be consistently introduced in ng-diamond. TEM observations show that in nt-diamond, a significant portion of the grains contains intersectional nanotwins, forming tweed-like pattern characteristic of the int-diamond (cf. Fig, 2 in ref. ${ }^{7}$ and cf. Supplementary Fig. 2 in ref. ${ }^{8}$ ). These observations suggest that int-diamond is readily manufacturable. The challenge is how to produce int-diamond consistently in bulk samples. Based on previous experimental results, two potentially important parameters to be grain size of the onion carbon precursors (highly deformed graphene layers in precursors increase the chance of TB formation in the diamond formation) and pressure and temperature conditions (high nucleation rates for diamond formation also increase the chance of producing TBs). Therefore, pre-deformation onion carbon precursors by uniaxial compression, large shear deformation ${ }^{38}$, and improvement of the synthesis pressure are a feasible method to form int-diamond in the experiment.

\section{DISCUSSION}

We have examined the mechanical properties of diamond with a novel microstructure by introducing intersectional twin boundaries in ng-diamond. A total of 39 slip systems in four slip modes of this designer diamond (int-diamond) are systematically analyzed. Based on the critical resolved shear stress of the four modes, we calculate the hardness of bulk int-diamond using the Sachs model and show that int-diamond is much harder than ntdiamond. The hardening mechanism of int-diamond is attributed to the intersectional TBs, which block dislocations motions, resulting in increased CRSS. These results are further confirmed by direct polycrystalline MD simulations. This work provides a new strategy for designing new super-hard materials in experiments.

\section{METHODS}

\section{Barrier strength}

The dislocation reaction with TB is a process of kink formation and migration, and it is schematically plotted in Supplementary Fig. 1. To simulate this process, a cuboid diamond twin structure model was first built. In this model, their $x, y$, and $z$ axis are along diamond matrix's [11 $\overline{2}$, [110], and [111] directions, the dimensions of $x, y$, and $z$ axis are 20.9, 2.5, and $13.8 \mathrm{~nm}$, respectively, and contains about 120000 carbon atoms. Next, a series of kinked shuffle-set $0^{\circ}$ and $60^{\circ}$ perfect dislocation with different kink pair widths was introduced in the twin plane of the cuboid diamond twin structure model by using dislocation displacement field method (in Supplementary Fig. 1) ${ }^{39,40}$.

MD simulations were then performed by using LAMMPS program ${ }^{41}$, and $\mathrm{C}-\mathrm{C}$ bonding interactions were described by LCBOP potential ${ }^{42}$. Periodic boundary condition was only imposed along the $y$ direction and the free surface was imposed in $x$ and $z$ directions. All these constructed structures were relaxed via energy minimization under different shear stress conditions. After relaxation, kink width dependent system energies were obtained and the maximum excess energy can be considered as kink formation energy $\left(2 E_{f}\right)$ at given shear stress. At the same time, a kink migration energy $\left(E_{m}\right)$ were calculated by using NEB method ${ }^{43}$. Finally, the activation energy $Q$ of dislocation reaction with TB is obtained according to $Q=2 E_{f}+E_{m}^{27}$. The shear stress-dependent activation energy for dislocation reaction with TB is plotted in Fig. 1d. As shown in Fig. 1d, when the activation energy of dislocation reaction with TB reaches zero, the corresponding shear stress can be considered as the barrier strength for a dislocation reacting with TB.

\section{Lattice friction stress}

For shuffle-set $0^{\circ}$ perfect dislocation slip in diamond, it also can be considered as a process of kink formation and migration, and the schematic for this process is plotted in insert of Supplementary Fig. 2. To simulate this process, a diamond structure model was built first. In this mode, its $x, y$, and $z$ axis are along the $[11 \overline{2}],[\overline{1} 10]$ and [111] direction and with dimensions of $20.9,2.5$, and $13.8 \mathrm{~nm}$, respectively. Then, a series of kinked shuffle-set $0^{\circ}$ perfect dislocation with different kink pair widths were introduced in the slip plane located at the center of the diamond structure model. On the basis of these models, the shear stress dependent kink formation and migration energy is obtained by adding shear stress to the diamond structure model by using the method as described above (methods section of Barrier strength). Finally, the shear stress-dependent activation energy of shuffle-set $0^{\circ}$ perfect dislocation slip in diamond is plotted in Supplementary Fig. 2. When the activation energy of the dislocation slip reaches zero, the corresponding stress is the lattice friction stress.

\section{int-diamond hardness by using Sachs model}

Sachs model is an effective method to investigate the yield strength for polycrystalline materials with anisotropic slip system. In Sachs model, the yield strength of each grain can be expressed as:

$$
\sigma_{n}=\min \left\{\sigma_{n}^{1}, \sigma_{n}^{2}, \sigma_{n}^{3} \ldots \ldots \sigma_{n}^{m}\right\},
$$

where the $\sigma_{n}^{m}$ represents the yield strength of $m$-th slip system in $n$-th grain and it can be expressed as following:

$\sigma_{n}^{m}=\frac{\tau_{m}^{\mathrm{CRSS}}}{\mu_{n}^{m}}$

where the $\tau_{m}^{\text {CRSS }}$ is the CRSS of $m$-th slip system; $\mu_{n}^{m}$ is the Schmid factor of $m$-th slip system in $n$-th grain.

In this work, a polycrystalline model with 6000 random orientations grains is considered. The yield strength for each grain can be obtained by using Eqs. 8 and 9. On the basis of these critical yield strength, we determine whether the grain yielded under a given uniaxial stress condition. As shown in Fig. 3a, the fraction of yielded grains increased with increasing uniaxial stress, when the fraction of yielded gain reaching $90 \%$, the corresponding stress can be considered as the yield strength for this polycrystalline material. Further its hardness can be obtained by tripling its yield strength ${ }^{33-35}$.

\section{MD simulation method for yield strength calculation}

In this work, atomic models for int-diamond, nt-diamond, and ngdiamond were constructed by using Voronoi polyhedron method ${ }^{44}$. As shown in Supplementary Fig. 3, each model contains 20 grains with an average grain size of $16.23 \mathrm{~nm}$. For int-diamond model, the twin boundaries $\mathrm{TB}_{1}$ have two types: $\Sigma 3(111)$ and $\Sigma 27(115)$ and twin boundary $\mathrm{TB}_{2}$ is $\Sigma 3(111)$. In this structure model, the fraction of twin boundary $\Sigma 3$ (111) is $\sim 75 \%$, and the fraction of twin boundary $\Sigma 3(111)$ can be further 
improved to asymptotically approach unity ${ }^{45}$. The twin thickness is $5.5 \mathrm{~nm}$ for $\mathrm{TB}_{1}$, and $1.2 \mathrm{~nm}$ for $\mathrm{TB}_{2}$. In the nt-diamond model, the twin thickness is 5.5 and $1.2 \mathrm{~nm}$.

The MD simulations were then performed on these atomic models by using the popular LAMMPS code ${ }^{41}$, and atomic configurations were visualized and analyzed by using the OVITO package ${ }^{46}$. In this MD simulation, the $\mathrm{C}-\mathrm{C}$ bonding interactions were described by Tersoff potential $^{47}$, and the isothermal-isobaric (NPT) scheme was used ${ }^{48}$. The time-step is set as $0.001 \mathrm{ps}$, relaxation time is $200 \mathrm{ps}$. After structure optimization under $300 \mathrm{~K}$ and ambient pressure, the compressive deformation is applied along $x$ direction under a constant strain rate of $5 \times 10^{8} \mathrm{~s}^{-1}$ with a total true strain of 0.3 , and the corresponding stressstrain curves were recorded. The maximum stress in the recorded stressstrain curves can be considered as the corresponding yield strength.

\section{DATA AVAILABILITY}

The authors declare that the data supporting the findings of this study are available within the paper and its Supplementary Information files.

\section{CODE AVAILABILITY}

All atomic simulations were performed by using the open source LAMMPS code $^{41}$.

Received: 28 May 2020; Accepted: 17 July 2020;

Published online: 07 August 2020

\section{REFERENCES}

1. Roundy, D. \& Cohen, M. L. Ideal strength of diamond, Si, and Ge. Phys. Rev. B 64, 212103 (2001)

2. Solozhenko, V. L. \& Godec, L. Y. A hunt for ultrahard materials. J. Appl. Phys. 126, 230401 (2019)

3. Brazhkin, V. V. \& Solozhenko, V. L. Myths about new ultrahard phases: Why materials that are significantly superior to diamond in elastic moduli and hardness are impossible. J. Appl. Phys. 125, 130901 (2019).

4. Irifune, T., Kurio, A., Sakamoto, S., Inoue, T. \& Sumiya, H. Ultrahard polycrystalline diamond from graphite. Nature 421, 599-600 (2003).

5. Sumiya, H. \& Irifune, T. Hardness and deformation microstructures of nanopolycrystalline diamonds synthesized from various carbons under high pressure and high temperature. J. Mater. Res. 22, 2345-2351 (2007).

6. Tang, $\mathrm{H}$. et al. Synthesis of nano-polycrystalline diamond in proximity to industrial conditions. Carbon 108, 1-6 (2016).

7. Huang, Q. et al. Nanotwinned diamond with unprecedented hardness and stability. Nature 510, 250-253 (2014).

8. Tao, Q. et al. Nanotwinned diamond synthesized from multicore carbon onion. Carbon 120, 405-410 (2017).

9. Yan, C. S. et al. Ultrahard diamond single crystals from chemical vapor deposition. Phys. Status Solidi (a) 201, R25-R27 (2004).

10. Hall, E. O. The Deformation and Ageing of Mild Steel_ III Discussion of Results. Proc. Phys. Soc. Sect. B 64, 747-753 (1951).

11. Petch, N. The cleavage strength of polycrystals. J. Iron Steel Inst. 174, 25-28 (1953).

12. Sumiya, H. \& Ishida, Y. Real hardness of high-purity ultra-fine nano-polycrystalline diamond synthesized by direct conversion sintering under HPHT. Diam. Relat. Mater. 100, 107560 (2019).

13. Boland, J. Science and nanotechnology of superhard materials. Natl Sci. Rev. 1, 4 (2014).

14. Xiao, J. et al. Dislocation behaviors in nanotwinned diamond. Sci. Adv. 4, eaat8195 (2018).

15. Tian, Y. et al. Ultrahard nanotwinned cubic boron nitride. Nature 493, 385-388 (2013).

16. Wen, B. et al. Continuous strengthening in nanotwinned diamond. npj Computational Mater. 5, 117 (2019).

17. Barnett, M., Keshavarz, Z. \& Ma, X. A semianalytical Sachs model for the flow stress of a magnesium alloy. Metall. Mater. Trans. A 37, 2283-2293 (2006).

18. Hirth, J. P. \& Lothe, J. Theory of dislocations, 2nd edn. (John Wiley and Sons, New York, 1982).

19. Jin, Z. H. et al. The interaction mechanism of screw dislocations with coherent twin boundaries in different face-centred cubic metals. Scr. Materialia 54, 1163-1168 (2006)
20. Wang, Y. et al. Strength and plastic deformation of polycrystalline diamond composites. High. Press. Res. 40, 35-53 (2020).

21. Zhang, H. W., Hei, Z. K., Liu, G., Lu, J. \& Lu, K. Formation of nanostructured surface layer on AISI 304 stainless steel by means of surface mechanical attrition treatment. Acta Materialia 51, 1871-1881 (2003).

22. Tao, N. R. \& Lu, K. Nanoscale structural refinement via deformation twinning in face-centered cubic metals. Scr. Materialia 60, 1039-1043 (2009).

23. Zhu, Y. T. et al. Dislocation-twin interactions in nanocrystalline fcc metals. Acta Materialia 59, 812-821 (2011).

24. $\mathrm{Ni}, \mathrm{S}$. et al. The effect of dislocation density on the interactions between dislocations and twin boundaries in nanocrystalline materials. Acta Materialia 60, 3181-3189 (2012).

25. Misra, A., Hirth, J. P. \& Hoagland, R. G. Length-scale-dependent deformation mechanisms in incoherent metallic multilayered composites. Acta Materialia 53, 4817-4824 (2005).

26. Lu, K. Stabilizing nanostructures in metals using grain and twin boundary architectures. Nat. Rev. Mater. 1, 16019 (2016).

27. Pizzagalli, L., Pedersen, A., Arnaldsson, A., Jónsson, H. \& Beauchamp, P. Theoretical study of kinks on screw dislocation in silicon. Phys. Rev. B 77, 064106 (2008).

28. Blumenau, A. T., Heggie, M. I., Fall, C. J., Jones, R. \& Frauenheim, T. Dislocations in diamond: core structures and energies. Phys. Rev. B 65, 205205 (2002).

29. Lu, L., Chen, X., Huang, X. \& Lu, K. Revealing the Maximum Strength in Nanotwinned Copper. Science 323, 607-610 (2009).

30. Chen, M. Deformation twinning in nanocrystalline aluminum. Science $\mathbf{3 0 0}$, 1275-1277 (2003)

31. Chen, K., Shi, S. Q. \& Lu, J. Tensile deformation properties of single crystal copper with nanotwins. Comput. Mater. Sci. 83, 269-276 (2014).

32. Taylor, G. I. Plastic strain in metals. J. Inst. Metals 62, 307-324 (1938).

33. Cahoon, J. R., Broughton, W. H. \& Kutzak, A. R. The determination of yield strength from hardness measurements. Metall. Trans. 2, 1979-1983 (1971).

34. Tiryakioğlu, M., Robinson, J. S., Salazar-Guapuriche, M. A., Zhao, Y. Y. \& Eason, P. D. Hardness-strength relationships in the aluminum alloy 7010. Mater. Sci. Eng.: A 631, 196-200 (2015).

35. Sekhar, A. P., Nandy, S., Ray, K. K. \& Das, D. Hardness-yield strength relation of AlMg-Si alloys. IOP Conf. Ser.: Mater. Sci. Eng. 338, 012011 (2018).

36. Ruoff, A. L. On the yield strength of diamond. J. Appl. Phys. 50, 3354-3356 (1979).

37. Gong, J., Wang, J. \& Guan, Z. A comparison between Knoop and Vickers hardness of silicon nitride ceramics. Mater. Lett. 56, 941-944 (2002).

38. Gao, Y. et al. Shear driven formation of nano-diamonds at sub-gigapascals and 300 K. Carbon 146, 364-368 (2019).

39. Barnett, D. M. The displacement field of a triangular dislocation loop. Philos. Mag. A 51, 383-387 (1985).

40. Barnett, D. M. \& Balluffi, R. W. The displacement field of a triangular dislocation loop-a correction with commentary. Philos. Mag. Lett. 87, 943-944 (2007).

41. Plimpton, S. Fast parallel algorithms for short-rang molecular dynamics. J. Computational Phys. 117, 1-19 (1995).

42. Los, J. H. \& Fasolino, A. Intrinsic long-range bond-order potential for carbon: Performance in Monte Carlo simulations of graphitization. Phys. Rev. B 68, 024107 (2003).

43. Izumi, S. \& Yip, S. Dislocation nucleation from a sharp corner in silicon. J. Appl. Phys. 104, 033513 (2008).

44. Voronoi, G. Nouvelles applications des paramètres continus à la théorie des formes quadratiques. J. f.ür. die reine und Angew. Mathematik 134, 198-287 (1908).

45. Gertsman, V. Y. \& Reed, B. W. On the three-dimensional twin-limited microstructure. Z. Fur Metallkd. 96, 1106-1111 (2005).

46. Stukowski, A. Visualization and analysis of atomistic simulation data with OVITO-the Open Visualization Tool. Model. Simul. Mater. Sci. Eng. 18, 015012 (2010).

47. Tersoff, J. Modeling solid-state chemistry: Interatomic potentials for multicomponent systems. Phys. Rev. B 39, 5566-5568 (1989).

48. Wood, W. W. Monte carlo calculations for hard disks in the isothermal-isobaric ensemble. J. Chem. Phys. 48, 415 (1968).

\section{ACKNOWLEDGEMENTS}

This work was supported by the National Natural Science Foundation of China (NSFC, Grant Numbers 51925105, 51771165 and 51525205).

\section{AUTHOR CONTRIBUTIONS}

B.W. conceived the project. J.X. performed all calculations. J.X and B.W. analyzed the calculated results. J.X., B.W., B.X., X.Z., Y.W., and Y.T. co-wrote the paper. All authors discussed the results and commented on the paper. 


\section{COMPETING INTERESTS}

The authors declare no competing interests.

\section{ADDITIONAL INFORMATION}

Supplementary information is available for this paper at https://doi.org/10.1038/ s41524-020-00387-3.

Correspondence and requests for materials should be addressed to B.W.

Reprints and permission information is available at http://www.nature.com/ reprints

Publisher's note Springer Nature remains neutral with regard to jurisdictional claims in published maps and institutional affiliations.
Open Access This article is licensed under a Creative Commons Attribution 4.0 International License, which permits use, sharing, adaptation, distribution and reproduction in any medium or format, as long as you give appropriate credit to the original author(s) and the source, provide a link to the Creative Commons license, and indicate if changes were made. The images or other third party material in this article are included in the article's Creative Commons license, unless indicated otherwise in a credit line to the material. If material is not included in the article's Creative Commons license and your intended use is not permitted by statutory regulation or exceeds the permitted use, you will need to obtain permission directly from the copyright holder. To view a copy of this license, visit http://creativecommons. org/licenses/by/4.0/.

(c) The Author(s) 2020 